\title{
EJNSO
}

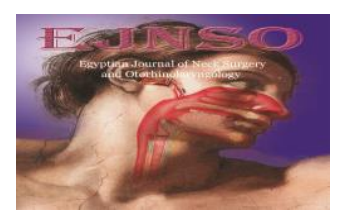

\section{Button graft and trichloroacetic acid for repair of small and medium-sized ear drum perforations}

\author{
Zaki F. Aref ${ }^{1}$ and Wael A. Ahmed ${ }^{2}$ \\ Otorhinolaryngology Department, South Valley University Hospital, Qena, Egypt \\ Otorhinolaryngology Department, Sohag University Hospital, Sohag, Egypt
}

\begin{abstract}
:
Introduction: Several techniques have been used for repairing tympanic membrane perforations starting from chemical cauterization of the edges of the perforation to the use of autologous graft materials such as temporalis muscle fascia, perichondrium and cartilage.

Aim: To present our approach of combined use of cartilage perichondrium graft (button graft) and chemical cauterization with trichloroacetic acid (TCA) for repair small and medium sized perforations.

Materials and Methods: In this study 80 patients with small and medium sized TM perforations included and divided into two groups. In the study group button graft (BG) combined with TCA cauterization was performed while conventional temporalis fascia graft (TFG) underlay myringoplasty was used in the control group. We compared the operative time, time needed for resuming usual physical activity, time needed for graft epithelialization and postoperative anatomical and functional restoration.
\end{abstract}

Results: Mean operative time was $13.70 \pm 2.30$ minutes in BG with TCA group and $42.40 \pm 6.45$ minutes in TFG group ( $\mathrm{P}$ value $<0.001$ ). Time needed for complete epithelization of the graft was $(12.00 \pm 1.50$ days $)$ in BG with TCA approach and (21.40 \pm 2.20 days) in TFG myringoplasty ( $\mathrm{P}$ value $<0.001$ ). There was no significant difference between both techniques as regard anatomical and functional restoration .

Conclusion: Combined use of cartilage perichondrium graft and chemical cauterization with TCA is an effective technique which decreases operative time, allow for rapid postoperative healing with comparable anatomical and functional results.

Key words: Button graft, ear drum, repair, perforations

\section{Introduction}

Myringoplasty is the procedure of closure of perforations of the tympanic membrane. The first surgical closure of tympanic membrane perforations using an autograft (a full thickness free skin graft) was performed by Berthold in $1878 .^{1}$
Since that time several surgical techniques and graft materials have been used. Temporalis muscle fascia, perichondrium and cartilage are the most commonly used grafts. ${ }^{2}$ In 1998, Eavey introduced butterfly cartilage graft myringoplasty (BCGM) in which he used a specially designed composite 
perichondrium cartilage graft from the tragus. $^{3}$

In 2013, Abdelghany presented a modification of cartilage myringoplasty and named it, button graft (BG), composite cartilageperichondrium graft, that is harvested from the tragus and fashioned to be fixed (buttoned) in the perforation through a transcanal approach. ${ }^{4}$

Promoting healing of the TM through cauterizing the edges of the perforation by a chemical material (silver nitrate or trichloroacetic acid TCA) is another approach that was adopted since long time, some authors used cauterization with TCA together with paper patch. ${ }^{5,6}$ The present study reports evaluates the combined use of chemical cauterization by TCA and Button graft in closing TM perforations compared to the conventional TFG underlay myringoplasty.

\section{Patients and Methods:}

\section{Study design:}

This study was conducted in the Department of E.N.T in Qena university hospital between 2015 and 2017. The present study complied with the ethical standards of the relevant regional and institutional guidelines on human studies and with Declaration of Helsinki. Written informed consent was signed from each participant or their guardians. Patients with small and medium sized TM perforations were included in this study for whom myringoplasty was done. In this study two main surgical techniques were used; Button cartilage (BG) combined with TCA cauterization through a transcanal approach was performed in one group while conventional temporalis fascia graft (TFG) myringoplasty through a postauricular approach was used in the other (control) group. Detailed history taking and thorough clinical examination was achieved in all cases. Pure tone audiometry was performed to measure the air-bone gap (ABG) at the frequency range $500-4000 \mathrm{~Hz}$ using Madsen Itera 901. Post-operative audiological evaluation was conducted three months after surgery.

Patients were selected from both genders and from different age groups with dry tympanic membrane perforation for more than 8-12 weeks and air bone gap not more than $40 \mathrm{~dB}$. Patients' data including demographics, site and size of perforation, preoperative and postoperative audiometric findings, and postoperative graft take rates were recorded.

\section{Surgical technique:}

All procedures were performed under general anesthesia and surgery was performed via transcanal approach for button graft and postauricular approach for temporalis fascia graft. After preparing, sterilizing and draping the ear to be operated, operative microscope was used for examining the perforation. TCA was used to cauterize edge of the perforation and adjacent area of the lateral surface of the tympanic membrane creating a denuded de-epithelialized circular area surrounding the perforation; about $2 \mathrm{~mm}$ of mucosa of medial surface of the tympanic membrane surrounding the perforation were also cauterized with TCA. A Fisch elevator was used for measuring the size of the perforation.

Tragal perichondrium-cartilage graft was harvested with the posterior perichondrium adherent and the anterior perichondrium left in the trageal area. The graft was designed in the form that the diameter of cartilage segment is smaller than the diameter of the adherent perichondrium layer. The perichondrium is separated from the 
peripheral $2 \mathrm{~mm}$ of the cartilaginous disc creating a groove that houses the margin of the perforation. Cartilage segment is also slightly larger $(2 \mathrm{~mm})$ than the size of the perforation while the perichondrium segment would overlay the perforation edges and the surrounding cauterized area on the lateral surface of TM.

The cartilage segment is buttoned through the perforation while the perichondrium is placed on the cauterized area of the lateral surface of TM.

Gelfoam is placed over the perichondrium and adjacent membrane rim and patient is discharged in the same day of surgery.

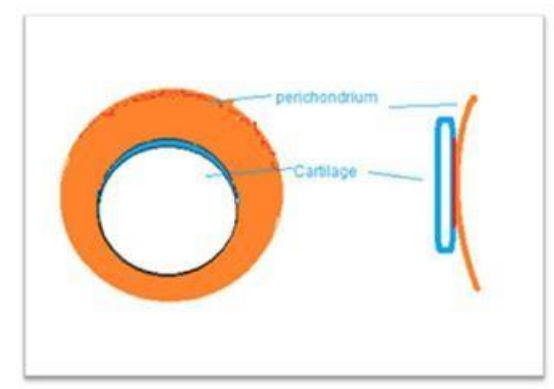

Fig 1. Diagram illustrating design of Button graft

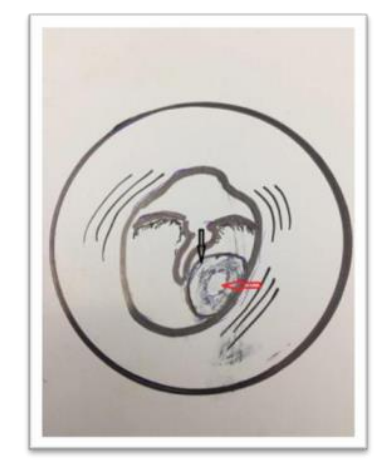

Fig 2. Diagram illustrating cauterization of the margins of the perforation with TCA. The black arrow points to the cauterized area while the red arrow points to the perforation.

\section{Statistical analysis:}

The data collected was analyzed by mean for quantitative variables; frequency and percentages were calculated for qualitative variables. Pearson Chi-Square test for comparing between both techniques. P-value of <
0.05 was established as significant. All analyses were carried out by SPSS version 19.

\section{Results:}

The present study included of 80 patients, 46 were males and 34 were females. The mean age of the study group was 29.6 years (range: 11- 40 years). Bilateral central perforation was found in 35 patients and unilateral perforation in 45 patients. Seventy-four patients had perforations due to infective etiology and 6 had post traumatic persistent perforations. Out of 80 patients, 40 underwent BG with chemical cauterization and 40 underwent TFG Myringoplasty. There was no significant difference between the mean ages of the two groups $(\mathrm{P}>$ 0.05 ). Mean operative time (excluding time for anesthesia) was $13.70 \pm 2.30$ minutes in BG with TCA group and 42.40 \pm 6.45 minutes in TFG group ( $P$ value $<0.001)$. Patients who underwent BG with TCA were advised to resume their usual activities significantly earlier than those who underwent TFG myringoplasty - table 1 .

Time needed for complete epithelization of the graft was significantly lesser in BG with TCA approach $(12.00 \pm 1.50$ days $)$ than in TFG myringoplasty (21.40 \pm 2.20 days) ( $\mathrm{P}$ value $<0.001)$.

Table (1): Comparison between BCGM with TCA and TFG myringoplasty

\begin{tabular}{|l|c|c|c|}
\hline \multirow{2}{*}{ Parameter } & $\begin{array}{c}\text { BCGM with } \\
\text { TCA }\end{array}$ & TFG & P-Value \\
\cline { 2 - 3 } & \multicolumn{2}{|c|}{ Mean \pm SD } & \\
\hline $\begin{array}{l}\text { Operative } \\
\text { timelmin }\end{array}$ & $13.70 \pm 2.30$ & $42.40 \pm 6.45$ & 0.000 \\
\hline $\begin{array}{l}\text { Duration of graft } \\
\text { epithelialization } \\
\text { day }\end{array}$ & $12.00 \pm 1.50$ & $21.40 \pm 2.20$ & 0.000 \\
\hline $\begin{array}{l}\text { Time for } \\
\text { resuming usual } \\
\text { activitylday }\end{array}$ & $3.00 \pm 0.70$ & $15.00 \pm 0.00$ & 0.000 \\
\hline
\end{tabular}




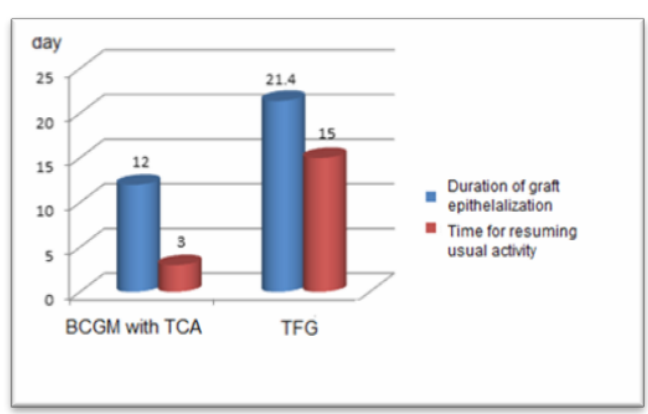

Fig 3. Difference between both techniques as regard operative time

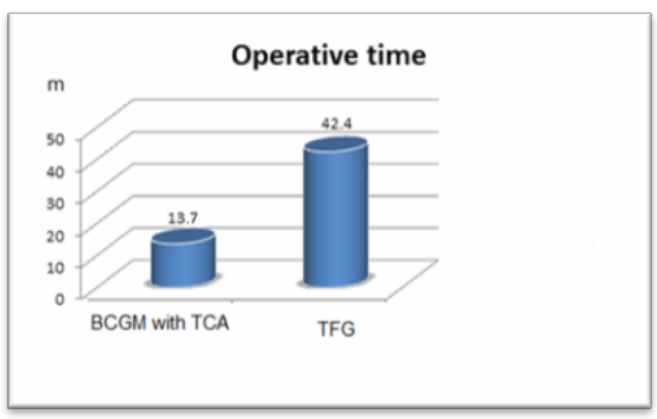

Fig 4. Difference between both techniques as regard time for resuming usual activities and duration of complete graft epithelialization
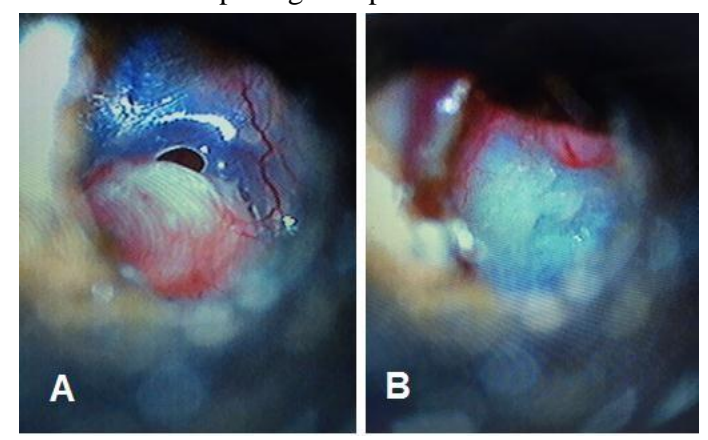

Fig 5. Microscopic view of the graft during application A. the cartilaginous segment is being inserted through the perforation, B. the cartilaginous segment is inserted inlay and perichondrium segment applied overlay.

Hearing restoration was calculated only for cases with successful grafting of the TM. Hearing improvement was observed by calculating the difference between mean preoperative and mean postoperative air bone gap. The difference between preoperative and postoperative $\mathrm{ABG}$ averages was $10{ }^{19}$ $\pm 4.27 \mathrm{~dB}(\mathrm{P}$ value $<0.001)$ in the study group and $10.53 \pm 4.59 \mathrm{~dB}(\mathrm{P}$ value <0.001) in the control group (TFG) with mean post-operative ABG
$6.78 \pm 4.12$ for BCG with TCA and $7.12 \pm 4.24$ for TFG myringoplasty.

\section{Discussion :}

Since it was first described by Eavy in 1998, butterfly cartilage tympanoplasty has gained popularity with many studies published about its efficiency. Following that technique, cartilage is fashioned to be inserted through TM perforation in the same was as a grommet tube without any tympanomeatal flap. ${ }^{3,7}$

A modified version of butterfly graft that allowed easier preparation and insertion of the graft (BG) was presented by Abdelghany in 2013 for managing small perforations under local anaethesia. ${ }^{4}$

Hosam et al in 2017 used plateletrich fibrin instead of TCA to increase success rate of graft uptake. ${ }^{8}$

In this study we present an additional step by using TCA for cauterizing the perforation margins and surrounding area of the lateral and medial surfaces of the tympanic membrane in order to prepare the tympanic membrane remnants and the perforation to adhere to the graft and induce rapid healing. The use of chemical cautery to promote healing of ear drum perforations has been used for long time. Chemical agents such as TCA induce an inflammatory response that stimulates healing. ${ }^{9} \mathrm{We}$ found that cautery with TCA is easy and rapid when compared to use of cold instruments for refreshing the margins of the perforation or de-epithelializing the membrane rim. We also expanded the use of BG to larger perforations up to $60 \%$ of the size of the TM.

Pfammatter et al (2013) has demonstrated a $90 \%$ success rate for closure of perforations of the ear drum which is the same result when compared to conventional TFG myringoplasty achieved through 
postauricular approach. Also, functional restoration measured by the improvement in air bone gab was similar in both groups and comparable to results reported in the literature. ${ }^{10}$

As regard the operative time, we found that BG with TCA is significantly time saving procedure with mean time of intervention approximately third that time needed for achieving conventional TFG myringoplasty. This time is also significantly lesser than mean time reported for butterfly cartilage myringoplasty. ${ }^{2,11}$

The duration of graft epithelialization was significantly lesser in the new technique than in conventional TFG myringoplasty. This may be related to the effect of TCA in promoting the process of healing. This advantage helps the patient to early resume his normal lifestyle early after the operation.

\section{Conclusion:}

Combined use of cartilageperichondrium graft and chemical cauterization with TCA is an effective way for repair of ear drum perforations which spares operative time and period of postoperative follow up with anatomical and functional results similar to the conventional TFG myringoplasty.

Conflict of interest: The authors declare no competing interests.

\section{Reference:}

1. Chalishazar U. Fat Plug Myringoplasty. Indian Journal of Otolaryngology and Head Neck Surgery, Vol. 57, No. 1, 2005, pp. 4344.

2. Özgür A, Dursun E, Terzi S, Erdivanlı ÖÇ, Coşkun ZÖ, Oğurlu M, Demirci M. Endoscopic butterfly cartilage myringoplasty. Acta Otolaryngol. 2016;136: 144-8.

3. Eavey RD. Inlay tympanoplasty: Cartilage butterfly technique. Laryngoscope 1998;108: 657-61.

4. Abdelghany AM. The button graft technique for perforations affecting less than $25 \%$ of the tympanic membrane: a non-randomised comparison of a new modification to cartilage tympanoplasty with underlay and overlay grafts. Clin Otolaryngol. 2013;38: 208-16.

5. Gulya AJ, Glasscock ME (2003) Glasscock, Shambaugh surgery of the ear, 5th edn. BC Decker Inc, Spain, pp 400-420

6. Santhi T, Rajan KV. A Study of Closure of Tympanic Membrane Perforations by Chemical Cauterisation. Indian Journal of Otolaryngology and Head \& Neck Surgery. 2012;64: 389- 392.

7.Eavey RD. Inlay tympanoplasty: cartilage butterfly technique. Laryngoscope 1998;108: 657-61.

8. Hosam M, Shaker M, Aboulwafa A. Effect of topical use of plateletrich fibrin in repairing central tympanic membrane perforation using the endoscopic inlay butterfly cartilage myringoplasty technique. The Egyptian Journal of Otolaryngology. 2017 Jul 1;33(3):557.

9. Park SN, Kim HM, Jin KS, Maeng JH, Yeo SW, Park SY. Predictors for outcome of paper patch myringoplasty in patients with chronic tympanic membrane perforations. Eur Arch Otorhinolaryngol. 2015;272: 297-301.

10.Pfammatter A, Novoa E, Linder T. Can myringoplasty close the air-bone gap? Otol Neurotol. 2013 Jun; 34:705-10.

11.Akyigit A, Karlidag T, Keles E, Kaygusuz I, Yalcın S, Polat C, Eroglu O. Endoscopic cartilage butterfly myringoplasty in children. Auris Nasus Larynx. 2017 Apr; 44:152-5. 